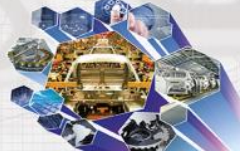

\title{
IMPLANTAÇÃO DE UM SENSOR VIRTUAL NO SISTEMA PIMS BASEADO EM REDES NEURAIS ARTIFICIAIS PARA FINS DE DIAGNÓSTICO DE PROCESSO*
}

\author{
Cláudio Antunes de Oliveira ${ }^{1}$ \\ Fernando da Silva Dummer² \\ João Marques Salomão ${ }^{3}$ \\ Hans Rolf Kulitz $z^{4}$
}

\section{Resumo}

O objetivo deste trabalho é utilizar o vasto banco de dados de informações de processo armazenadas pelo InfoPlus.21, que é um exemplo de sistema PIMS - Plant Information Managment System, para implementar um sensor virtual a partir de um modelo neural visando obter a tendência de resposta da variável consumo específico de uma caldeira. Propõe-se também, extrair diagnósticos do processo através do emprego da Lógica Fuzzy. As etapas de treinamento da rede neural e o desenvolvimento do diagnóstico envolveram simulações no software Matlab. Os resultados obtidos demonstraram-se bastante satisfatórios. Destaca-se a tendência de resposta do sensor virtual que apontou $97 \%$ de confiabilidade. Por fim entende-se que as propostas deste trabalho foram alcançadas, principalmente pela implementação inovadora do sensor virtual no InfoPlus.21 por meio da ferramenta SQLPlus, a qual possibilitará novas aplicações no meio industrial.

Palavras-chave: PIMS; Rede neural; Lógica fuzzy; Diagnóstico de processo.

\section{IMPLEMENTATION OF VIRTUAL SENSOR ON SYSTEM PIMS BASED ON NEURAL NETWORKS FOR PROCESS DIAGNOSIS}

\section{Abstract}

The propose of this work is to use the process information stored on InfoPlus.21 database to develop a virtual sensor from a neural model in order to obtain the variable trend. It is also proposed to extract the process diagnosis using the Fuzzy Logic concepts. All simulations to train the neural network, extract the neural model and develop the fuzzy diagnosis, were developed using Matlab software. The main result obtained was $97 \%$ reliability in the response of the virtual sensor. Finally, it is understood that proposals of this study were achieved, mainly by innovative implementation of the virtual sensor on InfoPlus.21 through SQLPlus tool, which will allow new applications in similar industrial cases.

Keywords: PIMS; Neural network; Fuzzy logic; Process diagnosis.

1 Engenheiro Eletricista, Especialização, Especialista em Automação, Gerência de Informática e Automação de Processo, ArcelorMittal Tubarão, Serra, ES e Brasil.

2 Engenheiro Eletricista, Especialista em Automação, Gerência de Informática e Automação de Processo, ArcelorMittal Tubarão, Serra, ES e Brasil.

3 Doutor em Engenharia Elétrica, Professor titular, Departamento de Engenharia Elétrica, Instituto Federal do Espírito Santo, Vitória, ES e Brasil.

4 Doutor em Engenharia Elétrica, Professor titular, Departamento de Engenharia Elétrica, Instituto Federal do Espírito Santo, Vitória, ES e Brasil.

* Contribuição técnica ao $18^{\circ}$ Seminário de Automação e TI Industrial, 23 a 26 de setembro de 2014, São Paulo, SP, Brasil. 


\section{INTRODUÇÃO}

Os sistemas PIMS - Process Information Management Systems são sistemas que adquirem dados de processo de diversas fontes, os armazenam num banco de dados históricos e os disponibilizam através de diversas formas de representação [1]. Uma destas formas é mostrada na figura 1 onde é possível analisar tendências históricas para investigação de falhas. A partir do banco de dados histórico é possível empregar técnicas de modelagem visando extrair conhecimento sobre o comportamento do processo ou de uma variável de interesse. Dentre as técnicas de modelagem empregadas, podemos destacar a obtenção de modelos a partir das redes neurais artificiais.

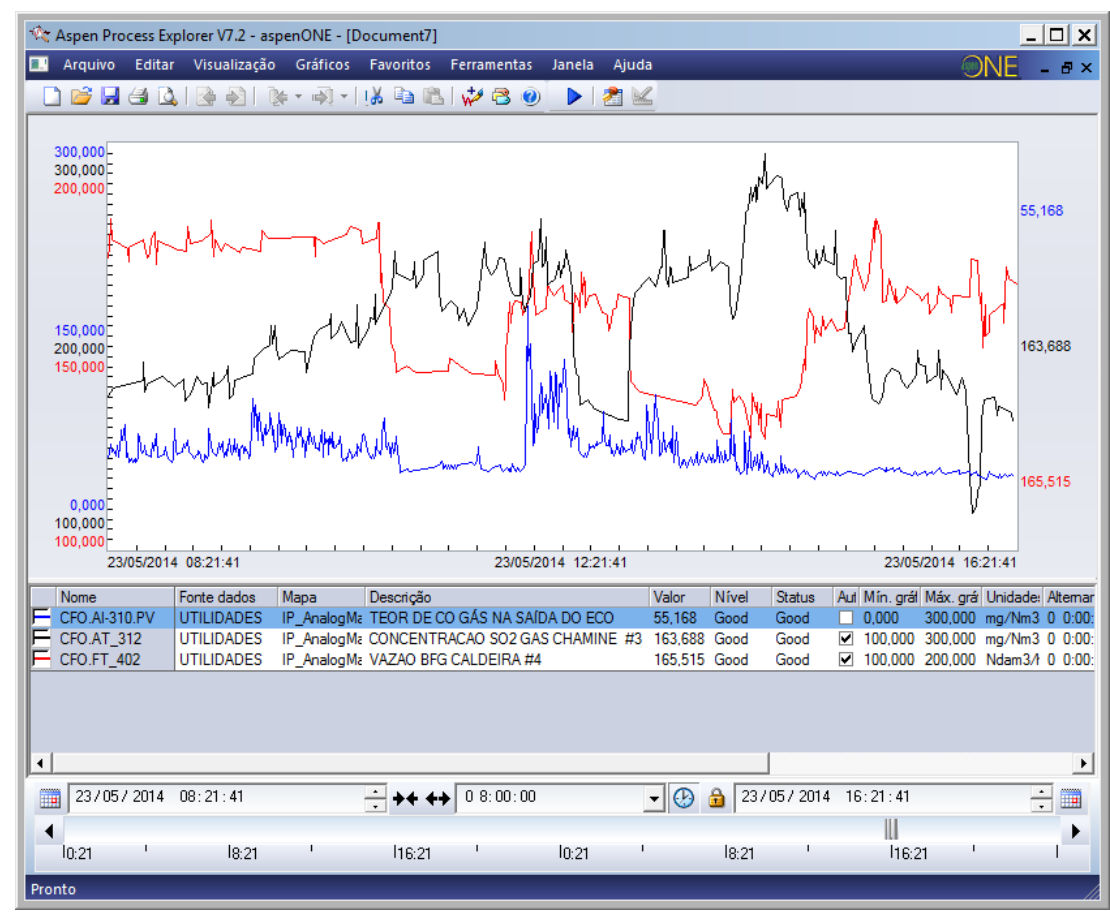

Figura 1 - Gráfico do InfoPlus.21

Redes Neurais Artificiais são técnicas computacionais que apresentam um modelo matemático inspirado na estrutura neural de organismos inteligentes e que adquirem conhecimento através da experiência. A operação de uma unidade de processamento, proposta por McCullock e Pitts em 1943, pode ser resumida da seguinte maneira:

- Sinais são apresentados à entrada;

- Cada sinal é multiplicado por um número, ou peso, que indica a sua influência na saída da unidade;

- É feita a soma ponderada dos sinais que produz um nível de atividade;

- Se este nível de atividade exceder um certo limite (threshold) a unidade produz uma determinada resposta de saída [2].

Um neurônio é uma unidade de processamento de informação que é fundamental para a operação de uma rede neural. O diagrama da figura 2 mostra o modelo de um neurônio.

\footnotetext{
* Contribuição técnica ao $18^{\circ}$ Seminário de Automação e TI Industrial, 23 a 26 de setembro de 2014, São Paulo, SP, Brasil.
} 

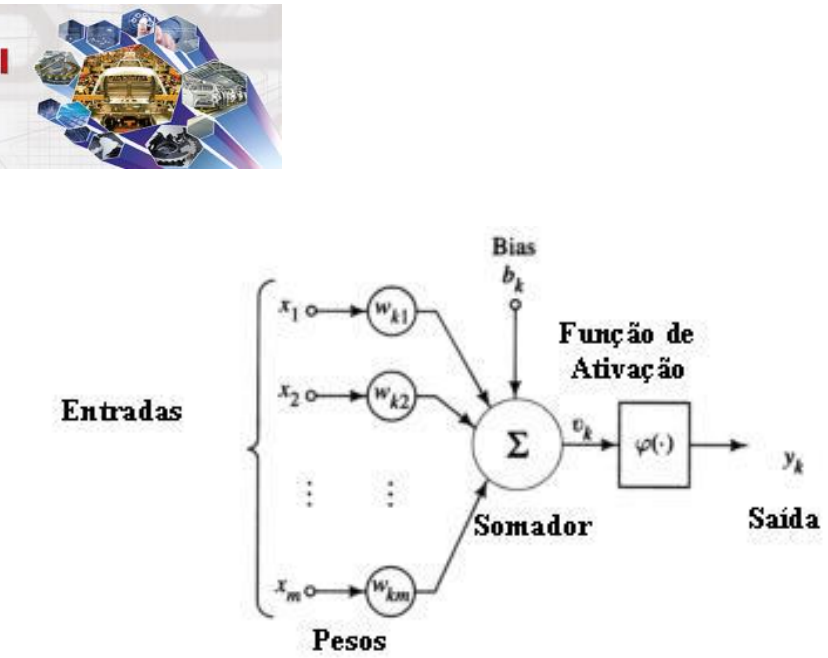

Figura 2 - Modelo de neurônio

Em termos matemáticos, podemos descrever um neurônio k escrevendo o seguinte par de equações:

e

$$
u_{k}=\sum_{j=1}^{m} w_{k j} x_{j}
$$

$$
y_{k}=\varphi\left(u_{k}+b_{k}\right)
$$

Onde $x_{1}, x_{2}, \ldots, x_{m}$ são os sinais de entrada; $w_{k 1}, w_{k 2}, \ldots, w_{k m}$ são os pesos sinápticos do neurônio $\mathrm{k}$; $u_{k}$ é a saída do combinador linear devido aos sinais de entrada; $b_{k}$ é o bias; $\varphi\left(\right.$.) é a função de ativação; e $y_{k}$ é o sinal de saída do neurônio [3].

Adotando-se esta técnica computacional, será abordado neste trabalho, o desenvolvimento de um sensor virtual bem com a sua implementação no InfoPlus.21, utilizando-se a ferramenta SQLPlus. O sensor virtual pode ser entendido como sendo uma medida não física, inferida em tempo real por meio de um modelo que descreva o comportamento do processo ou da variável física de interesse. Este modelo geralmente é obtido através de medidas físicas do processo que apresentam correlação com a variável que se deseja inferir e podem ser obtidos por diferentes tipos de técnicas. Há várias formas de se classificar técnicas de modelagem. Uma delas agrupa os métodos em três categorias denominadas modelagem caixa branca, modelagem caixa preta e modelagem caixa cinza [4].

O texto está organizado em 4 seções. A seção 2 descreve os materiais e métodos enquanto que os resultados são abordados na seção 3. Para finalizar, a seção 4 apresenta as conclusões e trabalhos futuros.

\section{MATERIAIS E MÉTODOS}

Este trabalho foi desenvolvido de acordo com as 6 etapas mostradas na figura 3, as quais serão detalhadas nos sub-tópicos seguintes. O software Microsoft Excel 2007 foi utilizado para coleta e tratamento dos dados e o software Matlab R2013a foi empregado para realização das simulações envolvendo a tanto a Rede Neural quanto a Lógica Fuzzy.

* Contribuição técnica ao 18Seminário de Automação e TI Industrial, 23 a 26 de setembro de 2014, São Paulo, SP, Brasil. 

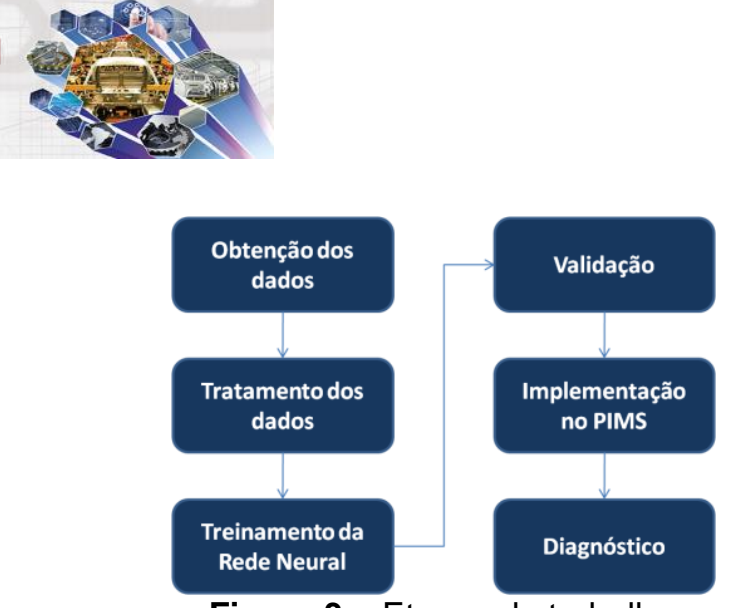

Figura 3 - Etapas do trabalho

\subsection{Obtenção dos Dados}

A massa de dados necessária para elaboração deste trabalho foi obtida de duas fontes distintas. A maior parte dela e abrangendo as variáveis de processo de entrada do modelo neural, foi obtida diretamente do InfoPlus.21, utilizando-se o recurso de exportar dados para o Microsoft Excel. Este recurso pode ser adicionado por meio da opção Suplementos do próprio Excel. Para complementar a massa de dados de treinamento, a variável de interesse do modelo neural foi extraída do Sistema de Otimização de Processo de Nível 2, onde esta variável é calculada. Assim, as 8 variáveis de processo mostradas na tabela 1, foram amostradas a uma freqüência de 60s por um período de 8 meses, totalizando uma massa de dados composta por 345.600 minutos.

Tabela 1 - Variáveis de entrada e saída

\begin{tabular}{c|lcc}
\hline Variável & \multicolumn{1}{|c}{ Descrição } & Unidade & Tipo \\
\hline 1 & Vazão de BFG - Blast Furnace Gas & $\mathrm{Nm}^{3} / \mathrm{h}$ & Entrada \\
2 & Vazão de COG - Coke Oven Gas & $\mathrm{Nm}^{3} / \mathrm{h}$ & Entrada \\
3 & Vazão de Alcatrão & $\mathrm{Nm}^{3} / \mathrm{h}$ & Entrada \\
4 & Vazão de Vapor & $\mathrm{Nm} 3 / \mathrm{h}$ & Entrada \\
5 & Poder calorífico inferior do COG & $\mathrm{Kcal} / \mathrm{m}^{3}$ & Entrada \\
6 & Poder calorífico inferior do BFG & $\mathrm{Kcal} / \mathrm{m}^{3}$ & Entrada \\
7 & Poder calorífico inferior do Alcatrão & $\mathrm{Kcal} / \mathrm{m}^{3}$ & Entrada \\
8 & Consumo específico da caldeira & - & Saída \\
\hline
\end{tabular}

\subsection{Tratamento dos Dados}

Para garantir um adequado processo de aprendizagem e/ou de treinamento da rede neural, o qual será abordado na próxima seção, foi necessário analisar a massa de dados coletada com o intuito de identificar condições atípicas de processo ou falhas nas medições que poderiam resultar em uma generalização incorreta da rede neural. Assim nesta etapa foram excluídos da massa de dados:

- Períodos de parada programada da planta;

- Problema de medição devido falha e/ou calibração de instrumento;

- Períodos em que a variável de interesse apresentou erro de cálculo no Sistema de Otimização de Processo de Nível 2.

\subsection{Treinamento da Rede Neural (Processo de Aprendizagem)}

Nesta etapa será abordado o treinamento da rede neural empregada neste trabalho, que é do tipo perceptron multicamadas. O algoritmo de treinamento utilizado foi o backpropagation. O objetivo desta etapa é a obtenção do modelo neural uilizando a

\footnotetext{
* Contribuição técnica ao $18^{\circ}$ Seminário de Automação e TI Industrial, 23 a 26 de setembro de 2014, São Paulo, SP, Brasil.
} 
ferramenta NNtool, a qual está disponível no software Matlab. A figura 4 mostra a tela principal desta ferramenta. Para acessá-la, basta digitar na linha de comando do Worspace do Matlab a palavra nntool e digitar Enter.

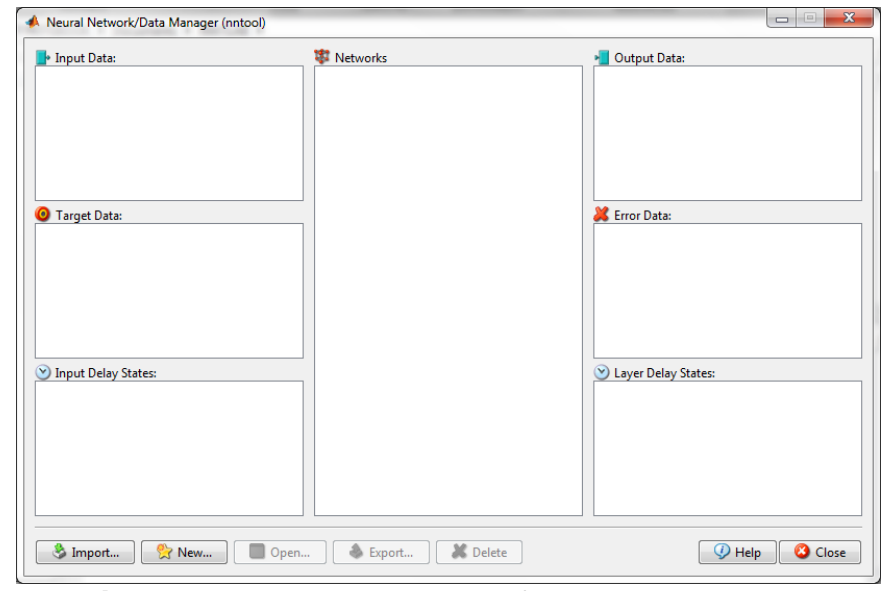

Figura 4 - Tela principal da ferramenta NNTool

De forma geral, necessitamos basicamente de duas operações para realizar o treinamento da rede neural através da ferramenta NNtool:

- Operação 1: através do botão Import efetuamos o carregamento das variáveis de entrada por meio do campo Input Data e posteriormente da variável de saída por meio do campo Target Data. Os dados de entrada e saída devem ser carregados primeiramente no Workspace do Matlab na forma de vetor transposto antes de iniciar a operação 1;

- Operação 2: uma vez carregados os dados de entrada e saída, defini-se os parâmetros da rede neural através do botão New. Os parâmetros configurados de forma mais usual são: o tipo de rede, o algoritmo de treinamento, o número de neurônios na camada escondida e o número de neurônios na camada de saída.

Após a configuração da rede neural é possível visualizar sua arquitetura por meio do botão Open a partir da tela principal da ferramenta NNtool. Uma nova tela será aberta e na guia View é possível visualizar a referida arquitetura. Na figura 5 é mostrada a arquitetura da rede neural utilizada na etapa de treinamento deste trabalho, a qual possui 4 blocos, descritos conforme segue:

- Input - um vetor contendo 7 variáveis de entrada;

- Hidden Layer - uma camada escondida composta por dois neurônios;

- Output Layer - uma camada de saída composta por um neurônio;

- Output - um vetor contendo 1 variável de saída.

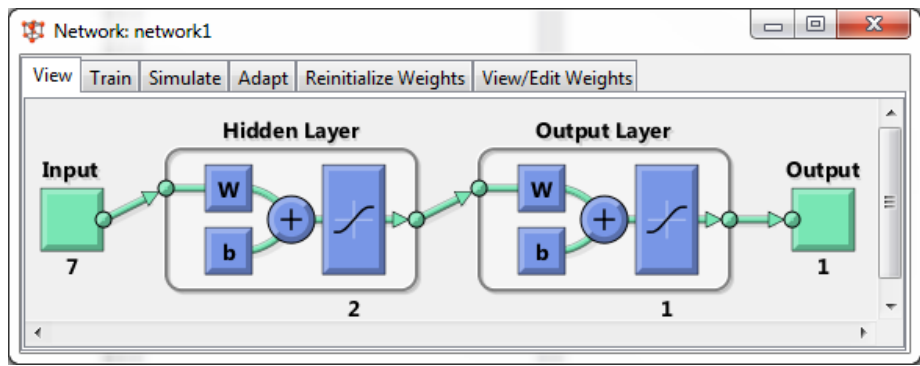

Figura 5 - Arquitetura da rede neural utilizada

* Contribuição técnica ao $18^{\circ}$ Seminário de Automação e TI Industrial, 23 a 26 de setembro de 2014, São Paulo, SP, Brasil. 
Uma vez que a arquitetura da rede neural está correta de acordo com a configuração realizada por meio das operações 1 e 2 citadas anteriormente, 0 treinamento propriamente dito da rede neural é realizado através da guia Train, conforme ilustrado na figura 6 . Nesta guia, basta informar novamente os vetores de entrada e saída nos campos Inputs e Targets respectivamente e clicar no botão Train Network.

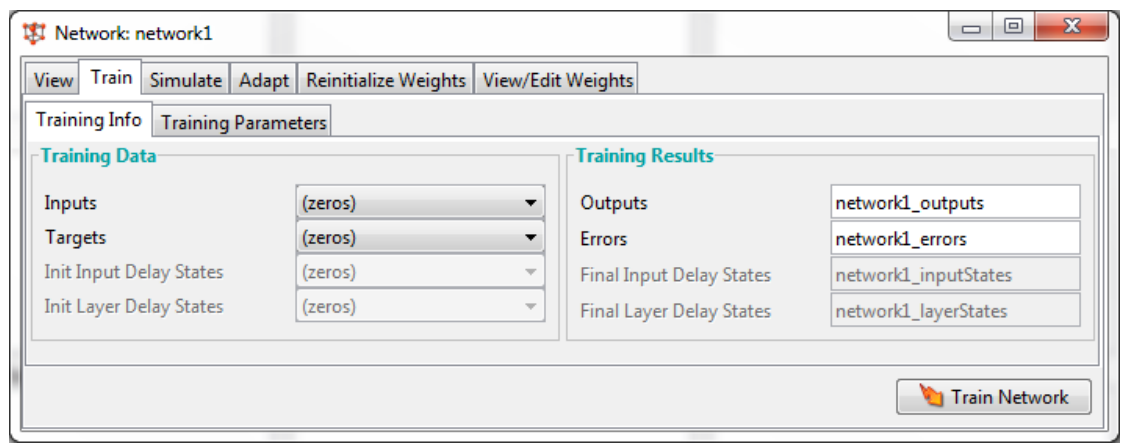

Figura 6 - Tela de treinamento da rede neural

Realizado o treinamento e este apresentado resultado satisfatório de acordo com o erro quadrático médio estipulado, podemos obter os parâmetros do modelo, os quais são descritos na tabela 2. Estes parâmetros são acessados através da guia View/Edit Weights, conforme figura 7.

Tabela 2 - Parâmetros do modelo neural

\begin{tabular}{l|c}
\hline \multicolumn{1}{c|}{ Parâmetro } & Descrição \\
\hline iw $\{1,1\}$ - Weight to layer 1 from input 1 & Pesos do neurônio 1 \\
iw $\{2,1\}$ - Weight to layer & Pesos do neurônio 2 \\
b\{1\}-Bias to layer 1 & Bias do neurônio 1 \\
b\{2\}-Bias to layer 2 & Bias do neurônio 2 \\
\hline
\end{tabular}

Estes parâmetros são utilizados no desenvolvimento do algoritmo que implementa o sensor virtual no InfoPlus.21. Este desenvolvimento será abordado no subitem seguinte.

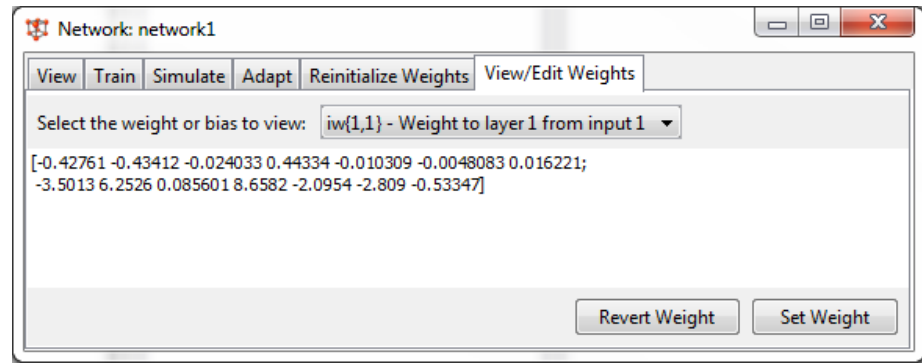

Figura 7 - Pesos da camada de entrada

\subsection{Implementação do Sensor Virtual no InfoPlus.21}

Utilizando o recurso da ferramenta SQLPLUS disponível no pacote de instalação do InfoPlus.21, foi desenvolvido um algoritmo em linguagem SQL para implementar o sensor virtual. $O$ algoritmo executa as 8 etapas mostradas na figura 8.

* Contribuição técnica ao $18^{\circ}$ Seminário de Automação e TI Industrial, 23 a 26 de setembro de 2014, São Paulo, SP, Brasil. 

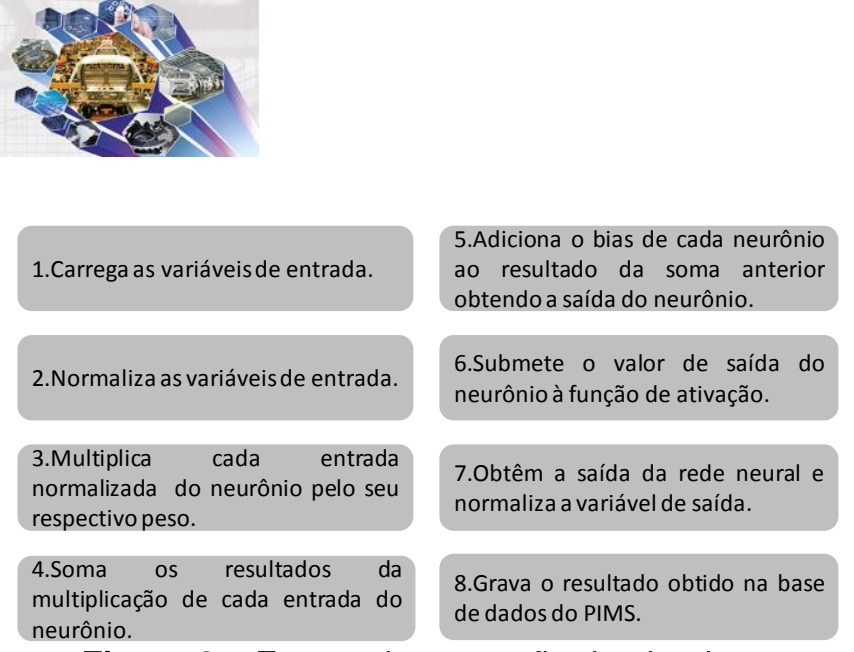

Figura 8 - Etapas de execução do algoritmo

A partir dos parâmetros de peso e bias obtidos após o treinamento da rede neural e conforme mostrado na tabela 2 do sub-item 2.3, foi implementado inicialmente no InfoPlus.21, a parametrização de 5 Queries, utilizando a opção QueryDef. As Queries criadas foram: IstBiasEntrada, IstBiasSaida, IstPesosEntrada, IstPesosSaida e IstMatrizEntrada. Um exemplo desta parametrização é mostrado na figura 9, onde podemos visualizar o arquivo IstBiasEntrada e o conteúdo da sua configuração efetuada a partir da opção QUERY_LINES. Nesta figura, os valores 3,9019 e 1,7342 correspondentes as linhas 3 e 4 respectivamente, são os parâmetros de bias de cada um dos dois neurônios da camada escondida.

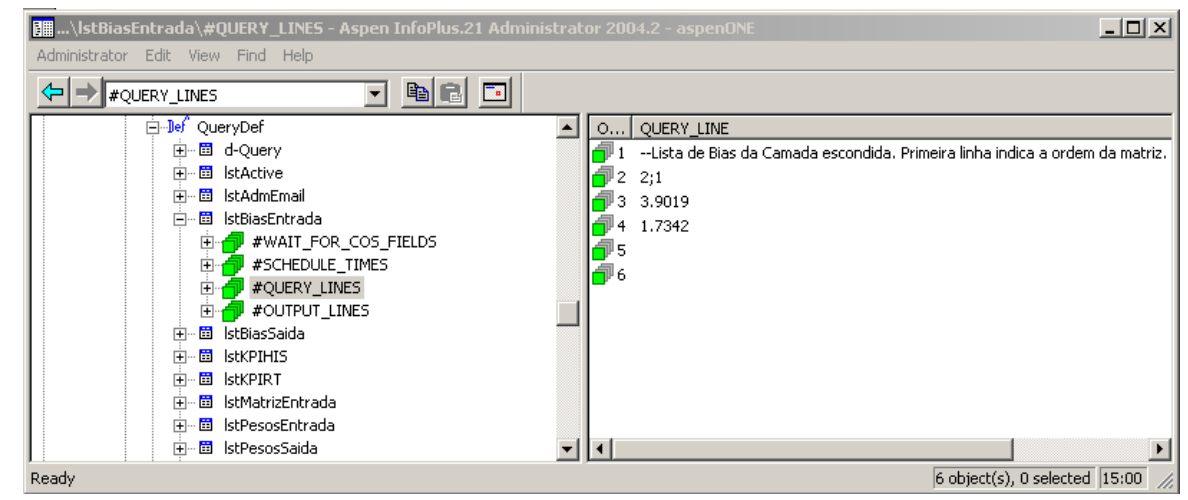

Figura 9 - Configuração da matriz IstBiasEntrada

Posteriormente foi elaborado o algoritmo do sensor virtual a partir da ferramenta SQLPlus. No início da sua execução, o algoritmo carrega cada uma das 5 Queries, pois estas contém os parâmetros do modelo neural obtido na etapa de treinamento. Um fragmento do algoritmo bem como o resultado de uma simulação executada a partir da ferramenta SQLPlus é mostrada na figura 10 e na figura 11 é mostrada um histórico da tendência de resposta do sensor virtual implantado no InfoPlus.21.

* Contribuição técnica ao 18Seminário de Automação e TI Industrial, 23 a 26 de setembro de 2014, São Paulo, SP, Brasil. 


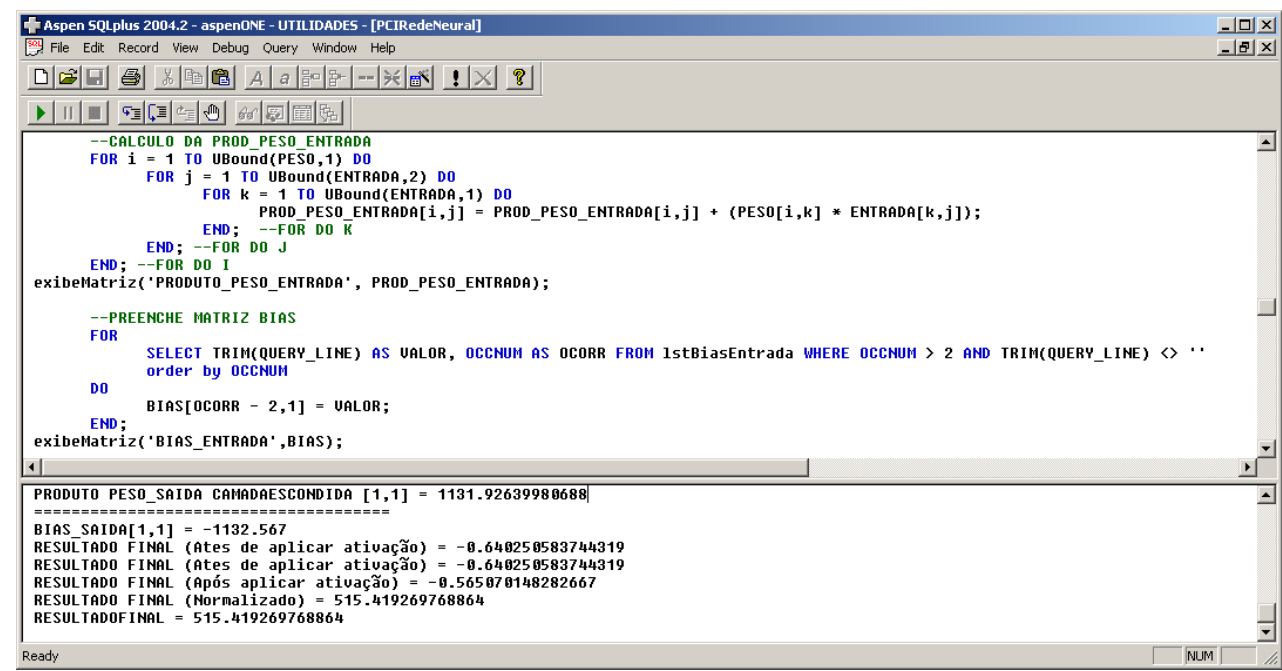

Figura 10 - Exemplo de simulação na ferramenta SQLPlus

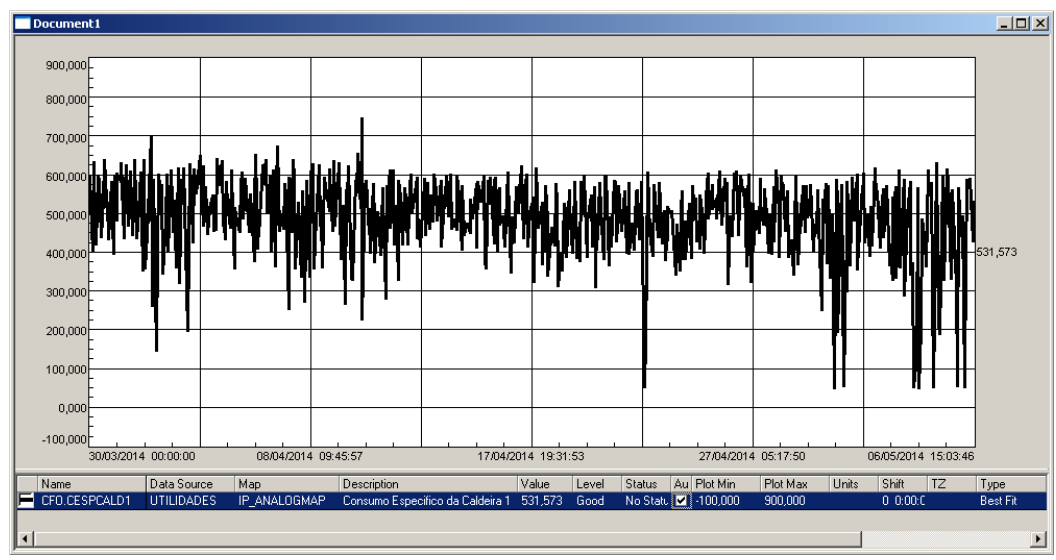

Figura 11 - Tendência de resposta do sensor virtual

\subsection{Validação do Algoritmo}

Nesta etapa de validação do algoritmo, comparou-se a resposta gerada pelo InfoPlus.21 com a resposta gerada pelo Matlab. Para isso, a rede neural obtida através da ferramenta NNTool, foi exportada para o Workspace do Matlab para permitir a obtenção da resposta deste sistema. Assim, por meio da função sim e de um conjunto aleatório de variáveis de entrada obtido diretamente do InfoPlus.21, determinou-se a resposta do Matlab. A tabela 3 mostra a comparação entre estas duas respostas.

Tabela 3 - Comparação entre as resposta do Matlab e do InfoPlus.21

\begin{tabular}{c|ccc}
\hline Amostra & Resposta Matlab & Resposta InfoPlus.21 & Erro \\
\hline 1 & 594,06 & 594,08 & 0,02 \\
2 & 599,99 & 600,01 & 0,02 \\
3 & 565,88 & 565,91 & 0,03 \\
\hline
\end{tabular}

Para complementar esta etapa de validação, comparou-se também a tendência de resposta do algoritmo implementado no InfoPlus.21 com a tendência da variável calculada no Sistema de Otimização de Processo de Nível 2. A figura 12 mostra a comparação destas duas respostas num período de 1 semana.

* Contribuição técnica ao 18Seminário de Automação e TI Industrial, 23 a 26 de setembro de 2014, São Paulo, SP, Brasil. 

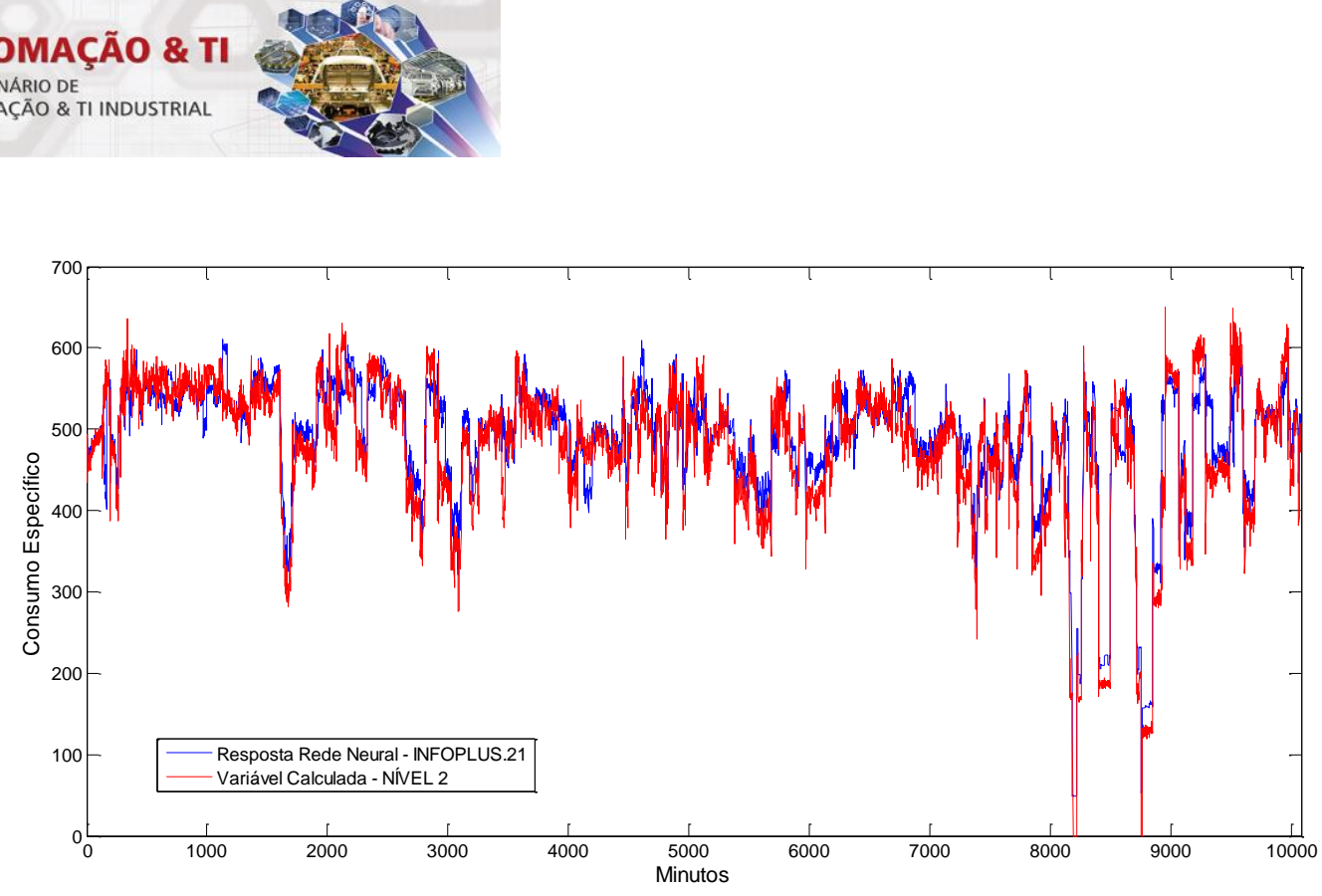

Figura 12 - Reposta do InfoPlus.21 e do Nível 2

\subsection{Diagnóstico}

O processo industrial escolhido para desenvolvimento deste sensor virtual foi uma caldeira, a qual faz parte de uma Central Termoelétrica. Neste tipo de processo, um dos principais indicadores é o consumo específico da caldeira, pois através dele, o operador determina suas ações de controle, visando maximizar a geração de vapor com menor consumo de combustível. Diante disto, propõe-se a utilização de outra técnica computacional - a Lógica Fuzzy, para inferir o diagnóstico deste processo.

A Lógica Fuzzy é uma técnica que incorpora a forma humana de pensar em um sistema de controle. Um controlador fuzzy típico pode ser projetado para comportarse conforme o raciocínio dedutivo, isto é, o processo que as pessoas utilizam para inferir conclusões baseadas em informações que elas já conhecem. Por exemplo, operadores humanos podem controlar processos industriais e plantas com características não-lineares e até com comportamento dinâmico pouco conhecido, através de experiência e inferência de relações entre as variáveis do processo. A Lógica Fuzzy pode capturar este conhecimento em um controlador fuzzy, possibilitando a implementação de um controlador computacional com desempenho equivalente ao do operador humano [5].

Adotando-se esta característica de inferência da Lógica Fuzzy, foi desenvolvida no Matlab uma proposta de diagnóstico do processo de combustão da caldeira, conforme ilustrado na figura 13. Como podemos perceber, as variáveis de entrada da máquina de inferência são as principais variáveis do processo de combustão da caldeira, acrescida da resposta do sensor virtual - o consumo específico da caldeira. A partir dessas entradas, a máquina de inferência, composta por um banco de regras que mapeia as condições de processo, ou seja, possui o conhecimento prévio do processo, gera o diagnóstico. A classificação da combustão por sua vez é inferida como: inadequada, regular e otimizada conforme mostrada na figura 14.

* Contribuição técnica ao 18Seminário de Automação e TI Industrial, 23 a 26 de setembro de 2014, São Paulo, SP, Brasil. 

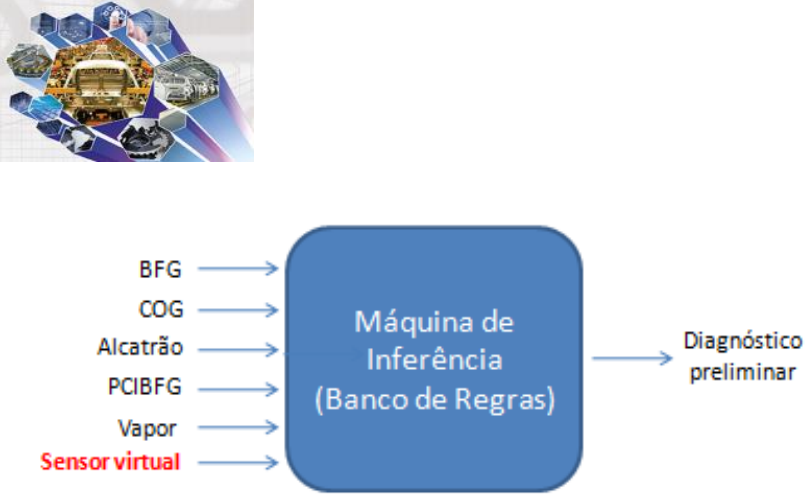

Figura 13 - Máquina de inferência fuzzy

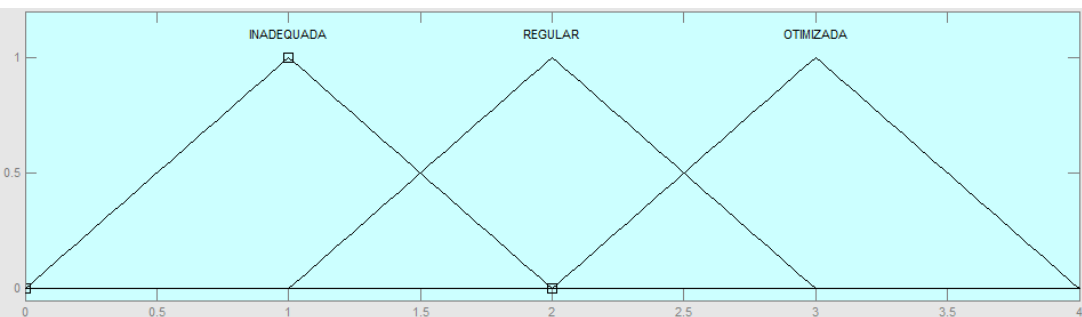

Figura 14 - Classificação do diagnóstico da combustão

\section{RESULTADOS E DISCUSSÃO}

Na figura 15 é mostrada a tendência de resposta do sensor virtual por um período de 30 dias. Como o interesse nesta aplicação é a tendência da variável consumo específico, inferida por meio do modelo neural, entende-se que o resultado alcançado foi bastante expressivo, o qual registrou $97 \%$ de confiabilidade na indicação do sensor virtual implementado no InfoPlus.21. Neste mesmo período, foi calculado o erro médio percentual entre a resposta do sensor virtual e o cálculo da variável de interesse realizado pelo Nível 2, o qual é apresentado na tabela 4.

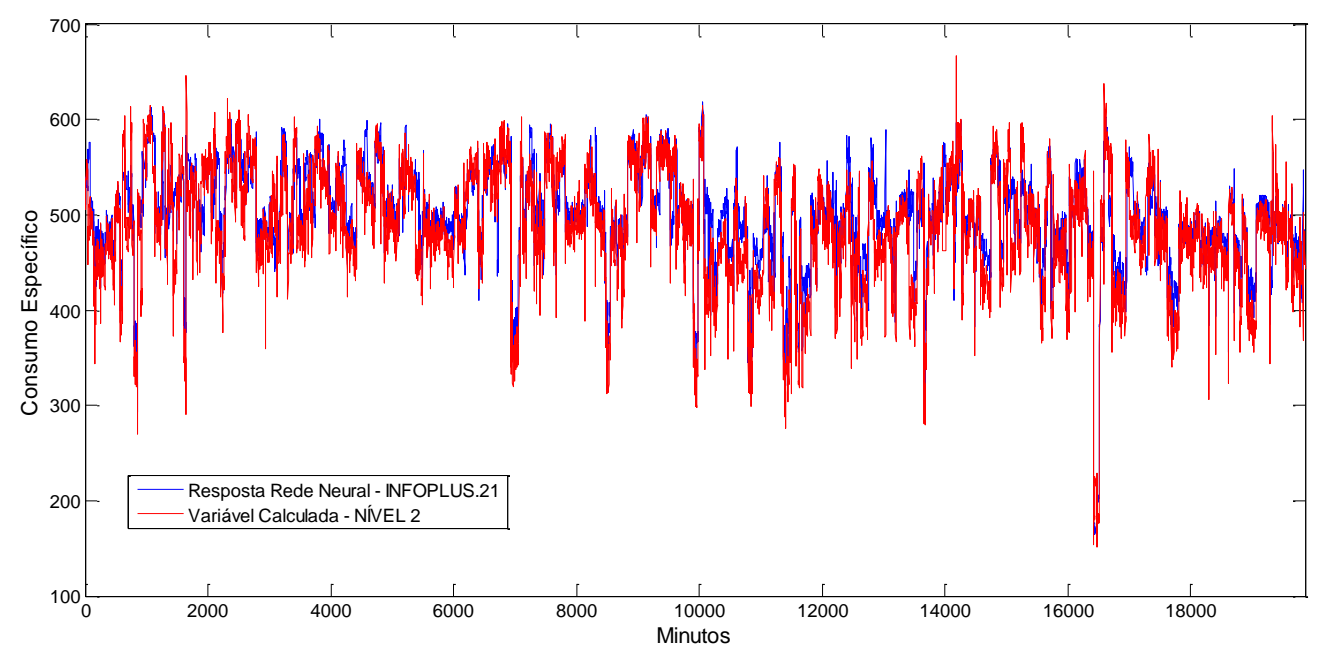

Figura 15 - Tendência de resposta do sensor virtual

Neste período, o erro médio percentual máximo foi verificado na semana 4, atingindo $-3,42 \%$. Vale ressaltar que este erro têm apresentado sinal negativo, apontando que em relação a variável calculada no Nível 2, a tendência de resposta do sensor virtual está menor. Levando-se em consideração a arquitetura da rede neural utilizada no treinamento, a qual utilizou apenas 2 neurônios na camada escondida, conforme abordado no subitem 2.3, sugere-se aumentar o número de neurônio desta camada, refazer a etapa de treinamento e avaliar o erro médio percentual. Porém, como o propósito é obter uma tendência da variável de interesse, optou-se por trabalhar com

* Contribuição técnica ao $18^{\circ}$ Seminário de Automação e TI Industrial, 23 a 26 de setembro de 2014, São Paulo, SP, Brasil. 
uma arquitetura mais enxuta, visando menor esforço computacional na implementação do sensor virtual no InfoPlus.21.

Tabela 4 - Erro médio apresentado pela rede neural

\begin{tabular}{c|ccc}
\hline Período & $\begin{array}{c}\text { Consumo Específico } \\
\text { (sensor virtual) }\end{array}$ & $\begin{array}{c}\text { Consumo Específico } \\
\text { (calculado) }\end{array}$ & $\begin{array}{c}\text { Erro Médio } \\
(\%)\end{array}$ \\
\hline Semana 1 & 506,65 & 517,56 & $-2,11$ \\
Semana 2 & 465,91 & 482,27 & $-3,39$ \\
Semana 3 & 478,29 & 490,40 & $-2,47$ \\
Semana 4 & 423,01 & 438,01 & $-3,42$ \\
\hline
\end{tabular}

Em relação ao diagnóstico proposto, as figuras 16 e 17 demonstram exemplos de resposta da máquina de inferência. A partir de condições reais de processo, 0 diagnóstico apresentado foi uma transição de otimizado para regular (o processo de combustão está com tendência de piorar) e otimizado, respectivamente.
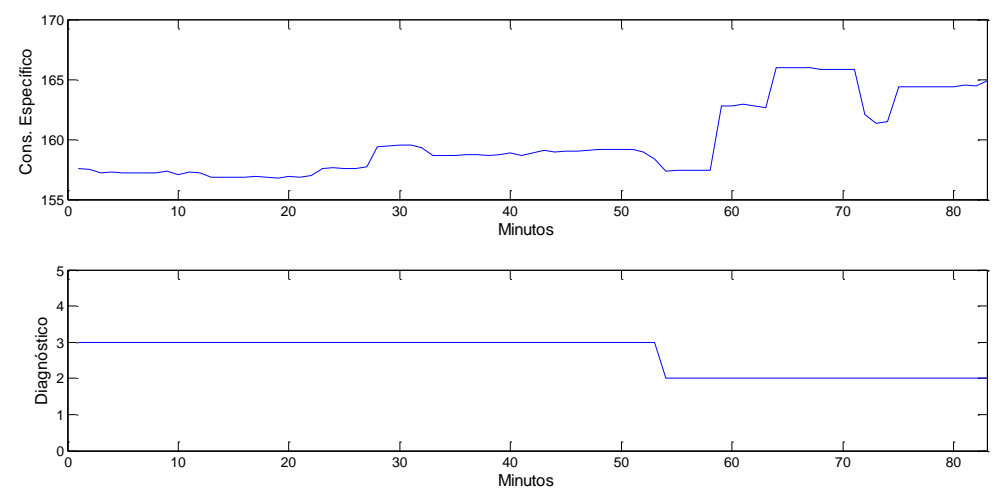

Figura 16 - Diagnóstico preliminar de transição
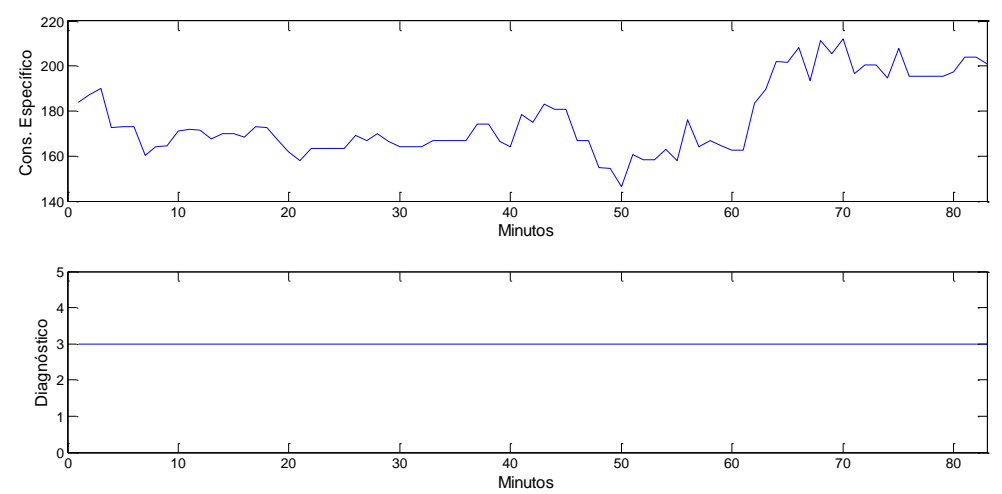

Figura 17 - Diagnóstico preliminar de combustão otimizada

\section{CONCLUSÃO}

Foi possível demonstrar neste trabalho, que as duas técnicas computacionais empregadas, demonstraram-se bastante aplicáveis em problemas de modelagem, onde a extração de conhecimento a partir de dados históricos de processo é de fundamental importância para a tomada de decisão do ponto de vista operacional.

Em relação à tendência de resposta do sensor virtual implantado no InfoPlus.21, considera-se que o resultado obtido de $97 \%$ de confiabilidade foi bastante satisfatório para a aplicação abordada neste trabalho, porém entende-se que

* Contribuição técnica ao 18Seminário de Automação e TI Industrial, 23 a 26 de setembro de 2014, São Paulo, SP, Brasil. 
melhorias na obtenção do modelo neural podem ser realizadas objetivando aumentar a confiabilidade do sensor virtual.

O diagnóstico proposto, embora tenha abordado apenas os combustíveis utilizados na caldeira, também apresentou bons resultados nas simulações. Porém como objeto de trabalho futuro, estuda-se a inclusão de novas regras na máquina de inferência, permitindo com isso enriquecer o diagnóstico proposto com outras condições de processo, além da implementação deste diagnóstico no Infoplus.21 ou no Sistema de Otimização de Processo de Nível 2.

Por fim, entende-se que o algoritmo desenvolvido no InfoPlus.21 permitirá alavancar novas aplicações de sensores virtuais. Dentre estas aplicações, pretende-se utilizar o banco de dados históricos do InfoPlus.21, para treinar uma rede neural preditiva visando obter antecipadamente o comportamento do processo.

\section{Agradecimentos}

Agradeço ao corpo docente do curso de Pós Graduação em Sistemas Inteligentes Aplicados a Automação do Instituto Federal do Espírito Santo, pelo conhecimento proporcionado, o qual possibilitou a base teórica necessária para desenvolver este trabalho. Agradeço também aos colegas da ArcelorMittal Tubarão do setor de Automação e das empresas parceiras que apoiaram este trabalho.

\section{REFERÊNCIAS}

1 PIMS Plant Information Managment System, Uma introdução. [acesso em 19 mar. 2014] 1-22. Disponível em:

http://www.das.ufsc.br/ rabelo/Ensino/DAS5316/MaterialDAS5316/Sistemas\%22MES\% 20MES\%20\&\%20PIMS/PIMS MES systems.pdf

2 Redes neurais artificais. [acesso em 11 abr. 2014] Disponível em: http://www.icmg.usp.br/pessoas/andre/research/neural/

3 Haykin S. Redes Neurais: princípios e prática. 2.ed. Porto Alegre: Bookman; 2001.

4 Aguirre LA. Introdução à Identificação de Sistemas: técnicas lineares e não lineares aplicadas a sistemas reais. 3.ed. rev. e ampl. Belo Horizonte: Editora UFMG; 2007.

5 Simões MG, Shaw IS. Controle e Modelagem Fuzzy. 2.ed. rev. e ampl. São Paulo: Blucher; 2007.

\section{BIBLIOGRAFIA}

1 Neural Network Toolbox, User's Guide. [acesso em 11 fev. 2014] Disponível em: http://www.mathworks.com/help/pdf_doc/nnet_ug.pdf

2 Fuzzy Logic Toolbox. [acesso em 11 fev. 2014] Disponível em: http://www.mathworks.com/products/fuzzy-logic/

* Contribuição técnica ao 18`Seminário de Automação e TI Industrial, 23 a 26 de setembro de 2014, São Paulo, SP, Brasil. 